\title{
Supersymmetry and the Atiyah-Singer Index Theorem*
}

\author{
Luis Alvarez-Gaumé $\star \star$ \\ Joseph Henry Laboratories, Princeton University, Princeton, NJ 08544, USA
}

\begin{abstract}
Using a recently introduced index for supersymmetric theories, we present a simple derivation of the Atiyah-Singer index theorem for classical complexes and its $G$-index generalization using elementary properties of quantum mechanical supersymmetric systems.
\end{abstract}

\section{Introduction}

The mathematical structure of supersymmetric field theories has recently been shown to be closely related to global questions of differential and algebraic geometry [1-3].

Given a supersymmetric quantum field theory, one can define a quantity, $\operatorname{Tr}(-1)^{F}$ (where $F$ is the fermion number) [1] which when properly regularized, counts the number of bosonic states minus the number of fermionic states in the Hilbert space of the theory. The properties of supersymmetry however, imply that this supersymmetric index depends only on the zero energy states due to the fact that all non-zero energy states appear in bose-fermi pairs. Furthermore, $\operatorname{Tr}(-1)^{F}$ is invariant under continuous deformations of the hamiltonian, and therefore is a topological index for the full quantum theory [1].

The fact that $\operatorname{Tr}(-1)^{F}$ is a topological index was used in [1] among other things to relate the possibility of supersymmetry breaking for supersymmetric $\sigma$-models to the vanishing of certain topological invariants of the manifold on which the $\sigma$-model is defined.

In this paper, we show that if we consider quantum mechanical supersymmetric systems (i.e., field theories in $0+1$ dimensions), and use $\operatorname{Tr}(-1)^{F}$ as a topological invariant, it is possible to calculate the index density for the Atiyah-

* Work supported in part by N.S.F. grants: PHY 82-15249, PHY 80-19754, PHY 82-03669, and the Harvard Society of Fellows

$\star \star$ Permanent address: Lyman Laboratory of Physics, Harvard University, Cambridge, MA 02138, USA 
Singer index theorem [4-6] for the classical complexes, and their corresponding $G$-index generalizations.

The organization of the paper is as follows: In Sect. II, we give a quick review of the properties of $\operatorname{Tr}(-1)^{F}$ that we will use. Section III presents the basic supersymmetric theory from which all the results can be obtained. Section IV contains the derivation of the index theorem for classical complexes. The conclusions are presented in Sect. V.

\section{Quantum Mechanics, Supersymmetry and $\operatorname{Tr}(-1)^{F}$}

The supersymmetry algebra in $0+1$ dimensions is defined by:

$$
\begin{aligned}
\left\{Q^{i}, Q^{* j}\right\} & =2 \delta^{i j} H, \quad i, j=1, \ldots, N, \\
\left\{Q^{i}, Q^{j}\right\} & =\left\{Q^{* i}, Q^{* j}\right\}=0,
\end{aligned}
$$

where $H$ is the hamiltonian of the system, and $Q^{i}, i=1, \ldots, N$ are the supersymmetric changes. Since in $0+1$ dimensions there is no spin, we have to define the fermion number operator $(-1)^{F}$ by requiring that it anticommutes with all the elementary fermion fields. For any of the $N$ charges in $(2.1)$ we have $(-1)^{F} Q_{i}$ $=-Q_{i}(-1)^{F}$. Since our arguments depend only on the existence of supersymmetric charges, we will concentrate on just one of the $Q^{\prime}$ 's. If instead of using $Q$ and $Q^{*}$, we define the real operator $\sqrt{2} S=Q+Q^{*}$, it follows that:

$$
S^{2}=H \text {. }
$$

Let $|E\rangle$ be an eigenstate of the hamiltonian $H$ with energy eigenvalue $E=0$. Then, acting with $S$ on $|E\rangle$ we get another eigenstate of $H, S|E\rangle$, with the same energy, and opposite fermion number with respect to $|E\rangle$, and the new state is nonvanishing because $E \neq 0$. Thus, all states with non-zero energy appear in FermiBose pairs, furnishing a two dimensional representation of supersymmetry for each energy level $E$. If we consider now the states with $E=0:|\Omega\rangle$, then $H|\Omega\rangle=0$, and $S|\Omega\rangle=0$; therefore, the zero energy states provide one dimensional representations of supersymmetry, and the number of zero energy bosonic states is not necessarily balanced by the number of fermionic ones. If we now compute $\operatorname{Tr}(-1)^{F}$, or the regularized trace, $\operatorname{Tr}(-1)^{F} e^{-\beta H}$, it follows that [1]:

$$
\operatorname{Tr}(-1)^{F} e^{-\beta H}=n_{B}^{E=0}-n_{F}^{E=0},
$$

where $n_{B}^{E=0}(F)$ counts the number of bosonic (fermionic) zero energy states. To show that $\operatorname{Tr}(-1)^{F}$ can also be understood as the index of an operator acting on the Hilbert space of the system, we go back to the description of $S$ in terms of $Q$ and $Q^{*}$. Splitting the Hilbert space into bosonic and fermionic subspaces: $\mathscr{H}=\mathscr{H}_{B} \oplus \mathscr{H}_{F}$, it follows that $S$ is an off-diagonal operator with respect to this splitting and that $Q|\psi\rangle=0, Q^{*}|\psi\rangle=0$ define the bosonic and fermionic zero energy states, respectively. Since $Q$ is the adjoint of $Q^{*}$ with respect to the Hilbert space norm, it follows that [1]:

$$
\operatorname{Tr}(-1)^{F}=\operatorname{Ker} Q-\operatorname{Ker} Q^{*} .
$$


Since the index of an operator is invariant under small continuous deformations, $\operatorname{Tr}(-1)^{F}$ is invariant under continuous changes of the parameters of the theory which do not change the asymptotic behavior of the hamiltonian for large values of the fields. Therefore, if in some reliable approximation we can calculate $\operatorname{Tr}(-1)^{F}$ perturbatively, we will obtain the index density for the operator $Q$.

It will be useful in what follows to realize that (2.3) has a functional integral representation [7]. In fact, $\operatorname{Tr}(-1)^{F} e^{-\beta H}$ is just the partition function at "temperature" $\beta^{-1}$ for an ensemble described by the density matrix $\varrho=(-1)^{F} e^{-\beta H}$. Standard arguments imply that

$$
\operatorname{Tr}(-1)^{F} e^{-\beta H}=\int_{\mathrm{PBC}} d \phi(t) d \psi(t) \exp -S_{E}(\phi, \psi),
$$

with the functional integral taken over field configurations satisfying periodic boundary conditions (PBC) with period $\beta$, and $S_{E}$ being the euclidean action of the theory.

The outline of our rederivation of the Atiyah-Singer results for classical complexes is as follows: We first find a supersymmetric quantum mechanical system whose conserved supercharge $Q$ is the operator whose index we want to calculate, and then we compute the $\beta$-independent term of the functional integral (2.5), which automatically yields the index density for the operator $Q$.

It is remarkable that the index theorem for the classical complexes (De Rahm, Dirac, signature and Dolbeault complexes) can be obtained from a single supersymmetric system, namely the supersymmetric non-linear $\sigma$-model. Furthermore, by a slight modification of the non-linear $\sigma$-model, it is possible to find as well the $G$-index theorem for the classical complexes.

\section{The Supersymmetric $\sigma$-Model}

The supersymmetric form of the $\sigma$-model can easily be obtained by finding the supersymmetric extension of the action for harmonic maps [8]. Let $\left(M_{n}, g\right)$ be an $n$-dimensional riemannian manifold and $g$ any smooth metric on it. Throughout this paper we will only consider riemannian connections on compact manifolds. The bosonic $\sigma$-model is defined by the Lagrangian:

$$
L=\frac{1}{2} g_{i j}(\phi) \dot{\phi}^{i} \dot{\phi}^{j}, \quad \dot{\phi}=\frac{d}{d t} \phi,
$$

where $\phi^{i}(t)$ are maps of $\mathbb{R}$ or $S^{1}$ onto the manifold $M$. The supersymmetric extension of (3.1) is [8]:

$$
\begin{gathered}
L=\frac{1}{2} g_{i j}(\phi) \dot{\phi}^{i} \dot{\phi}^{j}+\frac{i}{2} g_{i j}(\phi) \bar{\psi}^{i} \gamma^{0} \frac{D}{d t} \psi^{j}+\frac{1}{12} R_{i j k \ell} \bar{\psi}^{i} \psi^{k} \bar{\psi}^{j} \psi^{\ell}, \\
\frac{D}{d t} \psi^{i}=\frac{d}{d t} \psi^{i}+\Gamma_{j k}^{i} \dot{\phi}^{j} \psi^{k}, \\
\bar{\psi}_{\alpha}^{i}=\psi_{\beta}^{i} \gamma_{\beta \alpha}^{0}, \quad \alpha, \beta=1,2,
\end{gathered}
$$


$\psi^{i}=\left(\begin{array}{l}\psi_{1}^{i} \\ \psi_{2}^{i}\end{array}\right)$ is a two component real spinor, and $\gamma^{0}=\sigma_{2}$. The action (3.2) is invariant under the following supersymmetry transformations [8]:

$$
\begin{gathered}
\delta \phi^{i}=\bar{\varepsilon} \psi^{i}, \\
\delta \psi^{i}=-i \gamma^{0} \dot{\phi}^{i} \varepsilon-\Gamma^{i}{ }_{j k} \bar{\varepsilon} \psi^{j} \psi^{k},
\end{gathered}
$$

$\varepsilon$ being a real two component constant anticommuting spinor. In this section, we want to show that (3.2) contains all the ingredients necessary to compute the index density for the classical complexes. To see this, we first canonically quantize the theory. Shifting to a basis where $\gamma^{0}$ is diagonal, the canonical commutation relations for the fermions are given by:

$$
\left\{\psi^{i}, \psi^{* j}\right\}=g^{i j}(\phi), \quad\left\{\psi^{i}, \psi^{j}\right\}=\left\{\psi^{* i}, \psi^{* j}\right\}=0 .
$$

This implies that states without fermions are represented by functions on $M$, states with one fermion $\psi_{i}^{*}|\Omega\rangle$ correspond to one forms on the manifold and so on. The Hilbert space of the quantum theory is represented by the exterior algebra on $M: \Lambda^{*}(M)^{1}$. Using Noether's theorem to calculate the supersymmetric charges associated with (3.2), (3.3), one finds that $Q$ and $Q^{*}$ acting on $\Lambda^{*}(M)$ correspond identically to the exterior derivative and its adjoint, $d$ and $d^{*}$, so that the Hamiltonian is just the Laplacian on forms $H=d d^{*}+d^{*} d$. Hence the space of zero energy bosonic (fermionic) states is given by the set of even (odd) harmonic forms on $M_{n}$, and [1]

$$
\operatorname{Tr}(-1)^{F} e^{-\beta H}=\chi(M)=\sum_{k=0}^{n}(-1)^{k} b_{k},
$$

where $\chi(M)$ is the Euler number of $M$, and $b_{i}$ is the $i^{\text {th }}$ Betti number.

To obtain the Hirzebruch signature, it suffices to notice that (3.2) has a discrete symmetry $\psi_{\alpha}^{i} \rightarrow\left(\gamma_{5} \psi^{i}\right)_{\alpha}$, i.e. $\psi^{i} \rightarrow \psi^{* i}, \psi^{* i} \rightarrow-\psi^{i}$. If $Q_{5}$ is the operator implementing this symmetry, it easily follows that $\operatorname{Tr} Q_{5} e^{-\beta H}$ also depends only on the zero energy states. Geometrically, the action of $Q_{5}$ on $A^{*}(M)$ corresponds to the interchange of $\psi_{i}$ and $\psi_{i}^{*}$. Thus, it transforms $p$-forms into $n-p$ forms. Thus, $Q_{5}$ is the Hodge $*$-operation or Poincaré duality, and [1]

$$
\operatorname{Tr} Q_{5} e^{-\beta H}=\tau(M)=n^{E=0}\left(Q_{5}=+1\right)-n^{E=0}\left(Q_{5}=-1\right),
$$

where $\tau(M)$ is the Hirzebruch signature of $M$. Equation (3.6) is a bit different from (2.5), but it is also easy to obtain a functional integral representation for it. In this case, we have a "finite temperature" ensemble described by a density matrix $\varrho_{5}=Q_{5} e^{-\beta H}$. The functional integral is the same as in (2.5), but one has to integrate over periodic boundary conditions for the scalars $\phi^{i}$ and negative chirality spinors $\left(\gamma_{5} \psi^{i}=-\psi^{i}\right)$, and antiperiodic boundary conditions for positive chirality spinors $\left(\gamma_{5} \psi^{i}=+\psi^{i}\right)$.

The Dirac operator can be obtained by reducing (3.2) from $N=1$ to $N=\frac{1}{2}$ supersymmetry. What this means is that we consider the theory defined by (3.2)

1 To be precise, $\Lambda^{*}(M)$ is a $\mathrm{SO}(n)$ vector bundle over $M$. The space of wave function of our system is the space of sections of $\Lambda^{*}(M)$, i.e. $\Gamma\left(\Lambda^{*}(M)\right)$ 
and impose the condition $\psi_{1}^{i}=\psi_{2}^{i}=\psi^{i} / \sqrt{2}$. Then, the curvature term drops out due to the Bianchi identity, and the lagrangian reduces to

$$
L=\frac{1}{2} g_{i j}(\phi) \dot{\phi}^{i} \dot{\phi}^{j}+\frac{i}{2} g_{i j}(\phi) \psi^{i} \frac{D}{d t} \psi^{j}
$$

Using Noether's theorem again, it is easy to show that the supersymmetric charge one obtains os precisely the Dirac operator on the manifold $i \not D$. If the manifold admits a spin structure, we can compute (2.5) for (3.7) in order to obtain the index for the Dirac operator. If there is no spin structure, then something very similar to the SU(2)-anomaly occurs [9], and the functional integral is equal to zero due to the fact that the fermion pfaffian does not have a well defined sign.

Finally, to obtain the Dolbeault index (the index of $\bar{\partial}$ on a Kähler manifold [11]), we assume that the manifold $M_{n}$, on which the $\sigma$-model is defined, admits a Kähler structure. This simply means that the manifold can be covered with complex coordinate charts, with the transition functions being holomorphic, and with the line element satisfying

with

$$
d s^{2}=2 g_{\alpha \bar{\beta}} d z^{\alpha} d \bar{z}^{\beta},
$$

$$
\partial_{\alpha} g_{\lambda \bar{\mu}}=\partial_{\lambda} g_{\alpha \bar{\mu}}, \partial_{\bar{\alpha}} g_{\lambda \bar{\mu}}=\partial_{\bar{\mu}} g_{\lambda \bar{\alpha}},
$$

$\alpha, \bar{\alpha}=1, \ldots, n$ the complex dimension of the manifold, and $z^{\alpha}$ is a set of complex coordinates. Under these conditions, it is possible to show that the $\sigma$-model has two supersymmetries [10], and that the Kähler condition is both necessary and sufficient in order to have $N=2$ supersymmetry. Given the Kähler structure of the manifold, we can refine the exterior algebra $\Lambda^{*}(M)$ so that

$$
\Lambda^{*}(M)=\bigoplus_{p, q=0}^{n} \Lambda^{p, q}(M)
$$

$\Lambda^{p, q}(M)$ being the set of form which are $p$-times holomorphic and $q$-times antiholomorphic, i.e. $\Lambda^{p, q}(M)$ is generated by $d z^{\alpha_{1}} \wedge \ldots \wedge d z^{\alpha_{p}} d \bar{z}^{\beta_{1}} \wedge \ldots \wedge d \bar{z}^{\beta_{q}}$, with $\alpha_{1}<\ldots<\alpha_{p} \beta_{1}<\ldots<\beta_{q}$. This also implies that after canonical quantization, the fermionic creation (annihilation) operators can be divided up between those that create (annihilate) antiholomorphic or holomorphic indices. Hence, we can compute $\operatorname{Tr}(-1)^{F} e^{-\beta H}$ restricted to the Hilbert space sector which contain only antiholomorphic forms, i.e. we define

$$
\operatorname{Tr}^{\prime}(-1)^{F} e^{-\beta H}=\sum_{q=0}^{n} b^{0, q}(-1)^{q}=\operatorname{index}(\bar{\partial}) .
$$

Here $\operatorname{Tr}^{\prime}$ means that the trace is restricted to antiholomorphic forms; and $\partial, \bar{\partial}$ are such that if $\omega \in \Lambda^{p, q}, \partial \omega \in \Lambda^{p+1, q}$ and $\bar{\partial} \omega \in \Lambda^{p, q+1}$, so that $d=\partial+\bar{\partial}$.

The $G$-index theorems for the classical complexes can be obtained from a minor change of the $\sigma$-model (3.2). Let us assume that the manifold $M$ admits a Killing vector $K^{i}(\phi)$; then the following lagrangian is supersymmetric [12]:

$$
\begin{aligned}
L= & \frac{1}{2} g_{i j}(\phi) \dot{\phi}^{i} \dot{\phi}^{j}+\frac{i}{2} g_{i j}(\phi) \bar{\psi}^{i} \gamma^{0} \frac{D}{d t} \psi^{j}+\frac{1}{12} R_{i j k \ell} \bar{\psi}^{i} \psi^{k} \bar{\psi}^{j} \psi^{\ell} \\
& -\frac{\lambda^{2}}{2} g_{i j}(\phi) K^{i}(\phi) K^{j}(\phi)-\frac{\lambda}{2} \nabla_{i} K_{j} \bar{\psi}^{i} \gamma_{5} \psi^{j}, \gamma_{5}=\sigma_{z},
\end{aligned}
$$


and the supersymmetry transformations are:

$$
\begin{gathered}
\delta \phi^{i}=\bar{\varepsilon} \psi^{i} \\
\delta \psi^{i}=-i \gamma^{0} \dot{\phi}^{i} \varepsilon-\Gamma^{i}{ }_{j k} \bar{\varepsilon} \psi^{j} \psi^{k}-\lambda \gamma_{5} K^{i} \varepsilon .
\end{gathered}
$$

The supersymmetric algebra one obtains is different from (2.1) due to the presence of a central charge [18]. To see what this means geometrically, we again canonically quantize (3.12). The Hilbert space is still $\Lambda^{*}(M)$, but the action of the supersymmetry charge on $\Lambda^{*}(M)$ is not $d$, but rather $d_{\lambda}=d+\lambda i(\mathbf{K})$, where $i(\mathbf{K})$ is interior multiplication with the Killing vector $K$. (The $d_{\lambda}$ is one of the supersymmetry operators introduced in [2] to study the connections between supersymmetry and Morse theory.) Then, $d_{\lambda}^{2}=-d_{\lambda}^{* 2}=\lambda £(\mathbf{K}),[£(\mathbf{K})$ is the Lie derivative with respect to $\mathbf{K}$, and plays the role of the central charge] and $H_{\lambda}=d_{\lambda} d_{\lambda}^{*}+d_{\lambda}^{*} d_{\lambda}$. The zero energy states of $H$ are given by $d_{\lambda} \Omega=d_{\lambda}^{*} \Omega=0$, thus $£(\mathbf{K}) \Omega=0$, i.e., the zero energy states of $H$ are concentrated on $K$-invariant forms which are harmonic with respect to $d_{\lambda}, d_{\lambda}^{*}$ (in this subspace $d_{\lambda}^{2}=d_{\lambda}^{* 2}=0$ ). This result implies that if we compute $\operatorname{Tr}(-1)^{F} e^{-\beta H_{\lambda}}\left[H_{\lambda}\right.$ is the hamiltonian associated with (3.12)], we obtain the Lefschetz number of the De Rahm complex: $L(K)$.

In order to obtain the signature of $K$, notice that (3.12) has the discrete symmetry $\psi^{i} \rightarrow \sigma_{1} \psi^{i}, \sigma_{1}=\left(\begin{array}{ll}0 & 1 \\ 1 & 0\end{array}\right)$. Here $\sigma_{1}$ is essentially the same thing as Poincare duality, so, if we call $Q_{1}$ the operator producing this discrete symmetry, and repeat the arguments that led to the Hirzebruch signature, we obtain:

$$
\operatorname{Tr} Q_{1} e^{-\beta H_{\lambda}}=\operatorname{sign}(K)=L_{\text {sign }}(K) .
$$

Finally, repeating the arguments which led to the indices of the Dirac and Dolbeault complexes for the appropriate restrictions of (3.12), it follows that $\operatorname{Tr}(-1)^{F} e^{-\beta H_{\lambda}}$ gives their Lefshetz numbers with the proviso that $K$ must generate an orientation preserving spin isometry for the Dirac case or a holomorphic isometry for the Dolbeault complex.

The only thing left to obtain the index density for the operators of interest here is to evaluate their functional integral representations. This will be done in the next section.

To summarize, we have shown that the elliptic operators defined by the classical complexes are given either by the Hamiltonian or certain other symmetry operators of a $0+1$ dimensional supersymmetric field theory. This allows us to identify and interpret the index of the operators in field theoretic terms, namely, in terms of the ground states of the theory. Once this identification has been made, we can represent the index operator in a very compact form as a functional integral for the given field theory. This can be understood as a systematic and compact way of rewriting the computation of the index using the heat kernel of the relevant operator $[5,6]$. The fact that the field theory we have to use is supersymmetric is crucial in identifying the operators of interest in terms of a field theoretic Hamiltonian, in proving that the index will be invariant under continuous deformations, and in furnishing a systematic way of evaluating the index densities using standard methods in perturbation theory. 


\section{Computation of Index Densities}

We will start by deriving the Gauss-Bonnet formula for the Euler number. As was shown in the last section, this entails the computation of the functional integral (2.5) for the Lagrangian (3.2). In a basis where $\gamma^{0}$ is diagonal, (3.2) can be rewritten in the more convenient form:

$$
L=\frac{1}{2} g_{i j}(\phi) \dot{\phi}^{i} \dot{\phi}^{j}+i \psi^{* i} \frac{D}{d t} \psi^{j} g_{i j}-\frac{1}{4} R_{i j k \ell} \psi^{* i} \psi^{* j} \psi^{k} \psi^{\ell} .
$$

The integral (2.5) is over periodic configurations for the bosonic and fermionic fields, and it is independent of the parameter $\beta$; therefore we can expand $\phi, \psi$ in a Fourier series with frequencies $2 \pi n / \beta$. By making $\beta$ very small, the mass gap of the non-constant modes can be made arbitrarily large, so that the only strongly coupled modes are the constant configurations, while the non-constant modes can be treated on a perturbative basis. Thus the functional integral splits into an integral over constant bosonic and fermionic configurations (a finite dimensional integral) and an integral over non-constant configurations. The latter can be evaluated in perturbation theory, and its first term is given by the ratio of the fermionic and bosonic determinants on the gaussian approximation to the action for non-constant modes. The ratio of these determinants is 1 due to the supersymmetric Ward identities which imply that the zero point fluctuations of bosons and fermions cancel out exactly; put in a different way, the eigenvalues of the quadratic approximation to the action for both bosons and fermions are the same and with the same multiplicity. Thus in the small $\beta$-limit, and after rescaling the constant fermionic configuration by a factor of $\beta^{-1 / 4}$, we get:

$$
\operatorname{Tr}(-1)^{F} e^{-\beta H}=\frac{1}{(2 \pi)^{d / 2}} \int d(\mathrm{Vol}) \int \prod_{m} d \psi_{m}^{*} d \psi_{m} \exp -\frac{1}{4} R_{i j k \ell} \psi^{* i} \psi^{* j} \psi^{k} \psi^{\ell},
$$

where $d$ is the dimension of the manifold. If $d$ is odd, it is easy to see that $\operatorname{Tr}(-1)^{F} e^{-\beta H}=0$ because we cannot saturate the grassmann integrals with any term appearing in the expansion of the exponential. This is as it should be, because the Euler number of an odd dimension compact manifold is always zero. For the even case, it is trivial to show that:

$$
\begin{aligned}
\chi(M)= & \operatorname{Tr}(-1)^{F} e^{-\beta H}=\frac{(-1)^{d / 2}}{2^{d}\left(\frac{d}{2}\right) ! \pi^{d / 2}} \int d(\operatorname{Vol}) \varepsilon^{i_{1} j_{1} \ldots i_{n} j_{n}} \\
& \cdot \varepsilon^{k_{1} \ell_{1} \ldots k_{n} \ell_{n}} R_{i_{1} j_{1} k_{1} \ell_{1}} \ldots R_{i_{n} j_{n} k_{n} \ell_{n}}, d=2 n,
\end{aligned}
$$

which is the Gauss-Bonnet formula [13]. In the case of a four dimensional manifold, we get the well known result:

$$
\chi(M)=\frac{1}{32 \pi^{2}} \int_{M} \varepsilon_{a b c d} R_{a b} \wedge R_{c d}
$$

( $R_{a b}$ is the curvature 2-form in a vierbein basis).

To get the index density for other complexes requires some more work. Let us illustrate the basic ideas by computing the index density for the Hirzebruch 
signature. In this case we have to evaluate

$$
\tau(M)=\operatorname{Tr} Q_{5} e^{-\beta H}=\int_{\mathrm{PBC}}(d \phi)\left(d \psi_{2}\right) \int_{\mathrm{ABC}}\left(d \psi_{1}\right) \exp \left(-S_{E}\right) .
$$

Since fermions of the form $\left(\begin{array}{c}0 \\ \psi_{2}\end{array}\right)$ have $\gamma_{5}=-1$, they are integrated over with periodic boundary conditions (PBC) and fermions of the form $\left(\begin{array}{c}\psi_{1} \\ 0\end{array}\right)$ with $\gamma_{5}=+1$ are integrated over with antiperiodic boundary conditions $(\mathrm{ABC})$. In this case if we try to compute $\tau(M)$ as we did before for $\chi(M)$, we find that $\psi_{1}^{i}$ cannot have constant configurations due to the boundary conditions, so that for constant $\phi^{i}$ and $\psi_{2}^{i}$ and $\psi_{1}^{i}=0$ the action vanishes identically. This is not surprising, because these particular constant configurations are solutions to the classical equations of motion derived from (3.2) with zero action. Therefore, in order to compute the leading contribution to (4.5), we have to expand the lagrangian (3.2) around a constant background configuration $\left(\phi_{0}^{i}, \psi_{20}^{i}\right)$ up to second order in small fluctuations, evaluate the functional integrals in the gaussian approximation, and then integrate over all the constant configurations. The last step necessarily requires that we integrate only over non-constant small fluctuations in order to eliminate overcounting. Since the functional integral is invariant under coordinate reparameterizations of the manifold ( $\phi^{i}$ transform as a coordinate, and $\psi^{i}$ as a vector), we find it convenient to perform the expansion of the action $S(\phi, \psi)$ around constant background configurations in terms of normal coordinates. This can be done either by using superfield methods [14], or by expanding directly from the action (3.2) in terms of component fields. After a short computation, one obtains:

$$
\begin{aligned}
L^{(2)}= & \frac{1}{2} g_{i j}\left(\phi_{0}\right) \dot{\xi}^{i} \dot{\xi}^{j}+\frac{i}{2} \chi_{2}^{i} \frac{d}{d t} \chi_{2}^{j} g_{i j}\left(\phi_{0}\right)+\frac{i}{2} \psi_{1}^{i} \frac{d}{d t} \psi_{1}^{j} g_{i j}\left(\phi_{0}\right) \\
& +\frac{1}{4} R_{i j k \ell} \psi_{20}^{k} \psi_{20}^{\ell} \xi^{i} \dot{\xi}^{j}+\frac{1}{4} R_{i j k \ell} \psi_{20}^{k} \psi_{20}^{\ell} \psi_{1}^{i} \psi_{1}^{j},
\end{aligned}
$$

where $\xi^{i}$ and $\chi_{2}^{i}$ are small fluctuations around $\phi_{0}$ and $\psi_{20}$. We do not need to expand $\psi_{2}$ because there are no constant $\psi_{1}$ configurations and the action is automatically second order with respect to $\psi_{1}^{i}$. Finally, before writing down the result, we have to address the question of how to normalize the determinants that will appear after we integrate over $\xi, \chi$, and $\psi$. There are two ways of answering this question. The first is to notice that we are interested in the contributions which have a non-trivial dependence on the curvature, and therefore we lose nothing if we normalize with respect to the same determinants with the curvature set equal to zero. The second, and probably more rigorous, is to realize that for example the functional integral over $\psi_{1}^{i}$ is equivalent to computing the standard partition function at temperature $\beta^{-1}$ for a free gas of fermions moving in one dimension, with masses determined by the eigenvalues of the curvature (a similar argument applies also for the bosonic part of the functional integral); using elementary methods in statistical mechanics it is clear that the partition function calculated in this manner has no divergences and is free of normalization problems. Doing the computation by these two different methods, we get exactly the same answer, 
which is (after taking care of the factors of $i / 2 \pi$ arising from the Feynman measure in the functional integral):

$$
\tau(M)=\int_{M} d(\mathrm{Vol}) \int\left(d \psi_{20}\right) \prod_{\alpha} \frac{x_{\alpha}}{\tanh x_{\alpha}},
$$

where $x_{\alpha}$ 's are the skew eigenvalues of the curvature form

$$
\frac{1}{4 \pi} R_{a b c d} \psi_{20}^{c} \psi_{20}^{d}
$$

Formally, the constant anticommuting numbers $\psi_{20}^{a}$ are a realization of the basis of 1 -forms on the manifold, and the integral over $\psi_{20}^{a}$ just projects out the term proportional to $\psi_{20}^{1} \ldots \psi_{20}^{n}(n=\operatorname{dim} M)$ in the expansion of the integrand in (4.7). In a more geometrical way, let $\Omega_{a b}$ be the curvature two form, $\Omega_{a b}=R_{a b c d} e^{c} \Lambda e^{d} / 2$. For a riemannian manifold, $\Omega_{a b}$ is a 2 -form taking values in the Lie algebra of $\mathrm{SO}(n)$; if we formally skew-diagonalize $\frac{1}{2 \pi} \Omega_{a b}$, and label the eigenvalues again by $x_{\alpha}$, the polynomial :

$$
L(M) \equiv \prod_{\alpha} \frac{x_{\alpha}}{\tanh x_{\alpha}}
$$

defined by its power series expansion, defines a characteristic polynomial of the frame bundle over $M[15,16]$. Since $x_{\alpha}$ is a 2 -form for all $\alpha, L(M)$ contains only a finite number of terms, the highest order one (the "top form"), being proportional to the volume form of the manifold. The $L(M)$ is known as the Hirzebruch polynomial, and what we have shown is that the index of the signature complex is given by the top form of the Hirzebruch polynomial. In the specific case of 4-dimensional manifolds,

$$
\tau(M)=-\frac{1}{24 \pi^{2}} \int \operatorname{Tr} \Omega \wedge \Omega=b_{2}^{+}-b_{2}^{-},
$$

$b_{2}^{+}\left(b_{2}^{-}\right)$is the number of harmonic self-dual (antiself-dual) 2-forms.

The computation of the index density for the Dirac operator is analogous to what has just been described with the difference that the background expansion around constant field configurations of the lagrangian (3.7) is given by (4.6) after setting $\psi_{1}=0$, so that the only contribution comes from the bosonic determinant. The characteristic polynomial one obtains in this case is:

$$
\hat{A}(M)=\prod_{\alpha} \frac{x_{\alpha} / 2}{\sinh \left(x_{\alpha} / 2\right)},
$$

and its top form gives the index density for the Dirac operator. The $\hat{A}(M)$ is known as the Dirac genus $[15,16]$. Restricting (4.11) to the 4-dimensional case, we find that ind $(i \not D)=-\tau(M) / 8$.

For the Dolbeault complex, we choose the manifold to be a Kähler manifold. If we restrict the computation of $\operatorname{Tr}(-1)^{F} e^{-\beta H}$ to the antiholomorphic part of the Hilbert space, the computation is exactly the same as for the Dirac index. In this 
case we only have to notice that the background expansion around constant configurations has to be done using normal coordinates adapted to the Kähler structure, and that the curvature 2-form, when expressed in terms of normal coordinates, takes values on the Lie algebra of $\mathrm{U}(n)(d=2 n$ being the dimension of the manifold), so that the U(1) part of the curvature will also contribute to the zero point energy of the bosonic "oscillators" appearing in the expansion of the action to second order. As in the Dirac case, the non-trivial contribution comes from the bosonic determinant, and after a short computation, we get that the index density of $\bar{\partial}$ is determined by the following characteristic polynomial (the Todd genus):

$$
\operatorname{td}(M)=\prod_{\alpha} e^{\omega_{\alpha} / 2} \frac{\omega_{\alpha} / 2}{\sinh \omega_{\alpha} / 2}=\prod_{\alpha} \frac{\omega_{\alpha}}{1-e^{-\omega_{\alpha}}},
$$

where the $\omega_{\alpha}$ 's are the eigenvalue of:

$$
\Omega_{\alpha \bar{\beta}}=\frac{i}{2 \pi} R_{\alpha \bar{\beta} \lambda \bar{\mu}} d z^{\lambda} \wedge d \bar{z}^{\mu}
$$

In the 4-dimensional case it is easy to obtain that index $(\bar{\partial})=(\chi(M)+\tau(M)) / 4$.

It is remarkable that the four characteristic polynomials just derived can be combined into a single formula, which is a particular form of the Atiyah-Singer index theorem applied to elliptic operators defined on $\mathrm{SO}(n)$ bundles over the manifold $M_{n}$. To see this, we can translate the previous results into a more geometrical language. In each situation, we have two $\mathrm{SO}(n)$ bundles over $M_{n}, E$, and $F$, and an operator transforming sections of $E$ into sections of $F$, so that the section of both $E$ and $F$ generate a representation (reducible or irreducible) of $\mathrm{SO}(n)$. The curvature of $E(F)$ is given by the standard curvature 2-form but considered now as taking values on the representation of the Lie algebra of $\mathrm{SO}(n)$ generated by $E(F)$. Let $x_{\alpha}$ be the eigenvalues of $\Omega / 2 \pi(\Omega$ is the curvature 2 -form acting on the bundle). Then the Chern character is defined by the characteristic polynomial (for more details and examples see [16]):

$$
\operatorname{ch}(E)=\sum_{\alpha} e^{x_{\alpha}} .
$$

Then the index of any of the four operators considered above can be written in the form [16]

$$
\operatorname{ind}(0)=\int_{M} \frac{\operatorname{ch}(E)-\operatorname{ch}(F)}{e(M)} \operatorname{td}(M) ; e(M)=\prod_{i} x_{i},
$$

where we have to pick out the top form on the integrand, and calculate the Chern character and the Todd genus for the corresponding complexified bundles. If the operator is $d, E$, and $F$ correspond to even and odd forms; for *, they are the selfdual and atiselfdual forms; for $\bar{\partial}$ they are the even and odd anti-holomorphic forms; and for $i \not D$, they are the positive and negative chirality spin bundles. If one wanted to obtain the general form of the Atiyah-Singer index theorem, one could proceed along the lines of the second part of [5], but this will not be done in this paper.

We shall now derive the $G$-index generalization of the index theorem for classical complexes. 
We first start by proving the Lefshetz theorem. Let $K(\phi)$ be a Killing vector of the riemannian manifold $(M, g)$, and let $M_{i} i=1, \ldots, m$ be the set of fixed points of $M$. In principle, each $M_{i}$ is a submanifold of $M$. From Sect. III, we know that:

$$
L(K)=\int_{\mathrm{PBC}}(d \phi)(d \psi) \exp \left[-S_{\lambda}(\phi, \psi)\right],
$$

where $S_{\lambda}$ is the euclidean action defined by (3.12). Since the leading term is independent of $\lambda$, we can take $\lambda$ very large and $\beta$ small. This implies that on a perturbative expansion, the leading contributions come from the fixed points of $K$ so that:

$$
L(K)=\sum_{i=1}^{m} \int_{M_{\imath} \mathrm{PBC}}(d \phi)(d \psi) \exp -S_{i}(\phi, \psi),
$$

where $S_{i}$ is the euclidean action of the standard $\sigma$-model (3.2) restricted to the submanifold $M_{i}$. Recalling the computation of $\chi(M)$ at the beginning of this section we obtain:

$$
L(K)=\sum_{i=1}^{m} \chi\left(M_{i}\right)
$$

For the other classical complexes, we first make the assumption that $K$ has non-degenerate isolated fixed points $\phi_{0}^{i(\alpha)}, \alpha=1, \ldots, m$. Then $\nabla_{i} K_{j}$ is an antisymmetric matrix of maximal rank. Since the manifolds we have to consider for the other three classical complexes are even dimensional, this implies that $\nabla_{i} K_{j}$ does not have a vanishing determinant. At the fixed points, the action of the isometry generated by $K$ is just an $\mathrm{SO}(n)$ rotation in the tangent space, and therefore it can be decomposed into products of commuting $2 \times 2$ rotations through angles $\theta_{a}^{(\alpha)}, a=1, \ldots, n / 2$ which are the skew eigenvalues of $\nabla_{i} K_{j}$. To understand what we are computing, let $\phi_{s}$ be the one parameter group of transformations generated by the Killing vector $K^{i}$, and $\phi_{s}^{*}$ its pull-back operation mapping $E_{1,2} \rightarrow E_{1,2}$, where $E_{1,2}$ are the two bundles over $M$ in which the elliptic operator under consideration is defined. If $H_{1}, H_{2}$ are cohomology groups of the elliptic complex, then the Lefshetz number of the given elliptic complex for $\phi_{s}$ is defined by: ${ }^{2}$

$$
L_{E}\left(\phi_{s}\right)=\operatorname{Tr}\left(\phi_{s}^{*} H_{1}\right)-\operatorname{Tr}\left(\phi_{s}^{*} H_{2}\right)
$$

or in a different way, $H_{1}, H_{2}$ provide representation spaces for the isometry group generated by $K$. Therefore $\operatorname{Tr}\left(\phi_{s}^{*} H_{i}\right), i=1,2$ just computes the character of the representation of the isometry group on the representation space $H_{i}$. Thus, in order to calculate $L_{E}\left(\phi_{s}\right)$ using the functional integral, it is only necessary to realize that the role of $s$ is played by $i \lambda$, because in the way we have defined the action (3.12) [the central charge, which is the generator of the isometry, is an antihermitean operator for the supersymmetric algebra defined by (3.12)]. Therefore, we first compute the functional integral representing $L_{E}\left(\phi_{s}\right)$ for real $\lambda$, and then analytically continue to imaginary values. This is a well defined operation, because

2 For more details and examples in $G$-index theorems, see [16] 
the bosonic and fermionic determinants appearing in the evaluation of the functional integral are entire functions of $\lambda$ in the complex $\lambda$-plane.

If we consider first $L_{\text {spin }}\left(\phi_{s}\right)$, we have to compute

$$
\int_{\mathrm{PBC}}(d \phi)(d \psi) \exp -\int_{0}^{\beta} d t\left(\frac{1}{2} g_{i j}(\phi) \dot{\phi}^{i} \dot{\phi}^{j}+\frac{i}{2} \psi^{i} \frac{D}{d t} \psi^{j} g_{i j}-\frac{\lambda^{2}}{2} K_{i} K^{i}-i \frac{\lambda}{2} \nabla_{i} K_{j} \psi^{i} \psi^{j}\right)
$$

Taking again $\lambda$ large and $\beta$ small, the functional integral is dominated by the contributions coming from the fixed point set of the Killing vector. Expanding around the fixed points to second order on small fluctuations, computing the determinants, and performing the analytic continuation on $\lambda$, we arrive at:

$$
L_{\mathrm{spin}}\left(\phi_{s}\right)=\sum_{\phi_{0}^{(\alpha)}} \prod_{a} \frac{i}{2 \sin \left(s \theta_{a}^{(\alpha)} / 2\right)} .
$$

A similar argument applies for $L_{\text {sign }}\left(\phi_{s}\right)$ and $L_{\text {Dolb. }}\left(\phi_{s}\right)$ [for $L \operatorname{sign}\left(\phi_{s}\right)$ we have to compute $\left.\operatorname{Tr} Q_{1} e^{-\beta H_{\lambda}}\right]$ and yields ${ }^{3}$

$$
\begin{aligned}
L_{\mathrm{sign}}\left(\phi_{s}\right) & =\sum_{\phi_{0}^{(\alpha)}} \prod_{a} i \cot \left(s \theta_{a}^{(\alpha)} / 2\right), \\
L_{\text {Dolb. }}\left(\phi_{s}\right) & =\sum_{\phi_{0}^{(\alpha)}} \prod_{a} \frac{i e^{i s \theta_{a}^{(\alpha) / 2}}}{2 \sin \left(s \theta_{a}^{(\alpha)} / 2\right)} .
\end{aligned}
$$

Equations (4.20)-(4.23) are the generalized fixed point theorems first derived by Atiyah and Bott [17].

The extension of these results to fixed point sets consisting of non-trivial submanifolds of $M$ is straightforward. The only difference is that at each fixed set we will have, apart from the contribution from the non-vanishing rotation angles of the Killing vector, a factor proportional to the index of the corresponding complex restricted to the fixed set manifold. Similarly, it is also easy to obtain the corresponding formuli for discrete, rather than continuous, isometries.

\section{Conclusions}

This paper has been intended to give a pedagogical derivation of the AtiyahSinger index theorem for classical complexes. The method used requires only a little mathematical background and a working knowledge of the properties of supersymmetric quantum mechanical systems. It is rather remarkable that simple supersymmetric systems : the $N=1$ and $N=\frac{1}{2}$ supersymmetric $\sigma$-models, can give us so much topological information about the topology of the manifold where it is defined.

Throughout this work we have been assuming that the manifold is compact and without boundary. We have not pursued the derivation of the boundary corrections to the index theorems, although it seems likely that simple extension of the methods used here may yield the index theorems for compact manifolds with

$3 \quad L_{\text {sign }}\left(\phi_{s}\right)$ was also derived in [2] using supersymmetry but with a different method 
boundary, and that similar methods to ours may prove helpful in deriving new mathematical results.

Acknowledgements. I am grateful to $\mathrm{E}$. Witten for encouragement and many valuable discussions. I would also like to thank A. Jaffe, T. Bałaban, E. Getzler, T. Parker, C. Taubes, R. Bott, D. Freedman, M. Perry, and P. Ginsparg for useful discussions. And I would like to thank the Princeton Theory Group for their hospitality.

\section{References}

1. Witten, E.: Constraints on supersymmetry breaking. Nucl. Phys. B 202, 253 (1982)

Singer, I.M., McKean, H.P., Jr.: Curvature $\varepsilon$ the eigenvalues of the Laplacian. J. Diff. Geom. 1, 43-69 (1967)

2. Witten, E.: Supersymmetry and morse theory. J. Diff. Geom. 17, 661 (1982)

3. Witten, E.: Holomorphic morse inequalities. Princeton Preprint 1982

4. Atiyah, M.F., Singer, I.M.: Index of elliptic operators. I. Ann. Math. 87, 485 (1968) Atiyah, M.F., Segal, G.B.: Index of elliptic operators. II. Ann. Math. 87, 531 (1968)

Atiyah, M.F., Singer, I.M.: Index of elliptic operators. III. Ann. Math. 87, 546 (1968) Atiyah, M.F., Singer, I.M.: Index of elliptic operators. IV. Ann. Math. 93, 119 (1971) Atiyah, M.F., Singer, I.M.: Index of elliptic operators. V. Ann. Math. 93, 139 (1971)

5. Atiyah, M.F., Bott, R., Patodi, V.K.: On the heat equation and the index theorem. Invent. Math. 19, 279 (1973)

6. Gilkey, P.B.: The index theorem and the heat equation. Boston: Publish or Perish Inc. 1974

7. Cecotti, S., Girardello, L.: Functional measure, topology, and dynamical supersymmetry breaking. Phys. Lett. 110B, 39 (1982)

8. di Vecchia, P., Ferrara, S.: Classical solutions in two dimensional supersymmetric field theories. Nucl. Phys. B 130, 93 (1977)

Witten, E.: Supersymmetric form of the non-linear $\sigma$-model in two dimensions. Phys. Rev. D 16, 2991 (1977)

Freedman, D.Z., Townsend, P.K.: Antisymmetric tensor gauge theories and non-linear $\sigma$-models. Nucl. Phys. B 177, 282 (1981)

9. Witten, E.: An SU(2) anomaly. Phys. Lett. B 117, 324 (1982)

10. Zumino, B.: Supersymmetry and Kähler manifolds. Phys. Lett. B 87, 203 (1979)

11. Lichnerowicz, A.: Global theory of connections and the holonomy group. Leyden: Noordhoof 1976

12. Alvarez-Gaumé, L., Freedman, D.Z.: Potential terms in two dimensional extended supersymmetry. Harvard Preprint

13. Chern, S.S.: On the curvature integrals in a Riemannian manifold. Ann. Math. 46, 674 (1942)

14. Alvarez-Gaumé, L., Freedman, D.Z., Mukhi, S.: The background field method in supersymmetric $\sigma$-models. Ann. Phys. 134, 85 (1981)

15. Milnor, J.W., Stasheff, J.D.: Characteristic classes. Princeton, NJ : Princeton University Press 1974

16. Eguchi, T., Gilkey, P.B., Hanson, A.J.: Gravitation, gauge theories, and differential geometry. Phys. Rep. 213 (1980)

17. Atiyah, M.F., Bott, R.: A Lefshetz fixed point formula for elliptic differential operators. Bull. Am. Math. Soc. 12, 245 (1966)

Atiyah, M.F., Bott, R. : A Lefshetz fixed point formula for elliptic complexes. I, II. Ann. Math. 86, 374 (1967); 88, 451 (1968)

18. Haag, R., Lopuszanski, J., Sohnius, M.: All possible generators of supersymmetries of the $S$-matrix. Nucl. Phys. B 88, 61 (1975)

Communicated by A. Jaffe

Received April 14, 1983; in revised form May 9, 1983 
